\title{
Archéopages
}

Archéopages

Archéologie et société

40 | 04-07/2014

Villages

\section{Regard archéologique sur les villages martyrs de la Grande Guerre en Lorraine}

\section{Stéphanie Jacquemot}

\section{(2) OpenEdition}

12 Journals

Édition électronique

URL : https://journals.openedition.org/archeopages/641

DOI : $10.4000 /$ archeopages.641

ISSN : 2269-9872

\section{Éditeur}

INRAP - Institut national de recherches archéologiques préventives

\section{Édition imprimée}

Date de publication : 1 juin 2015

Pagination : 140-147

ISSN : $1622-8545$

\section{Référence électronique}

Stéphanie Jacquemot, «Regard archéologique sur les villages martyrs de la Grande Guerre en Lorraine », Archéopages [En ligne], 40 | 04-07/2014, mis en ligne le 01 juillet 2016, consulté le 21 janvier 2022. URL : http://journals.openedition.org/archeopages/641 ; DOI : https://doi.org/10.4000/ archeopages.641 


\section{Regard archéologique sur les villages martyrs de la Grande Guerre en Lorraine}

Stéphanie Jacquemot Ministere de la culture et de la communication

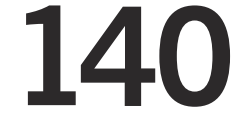

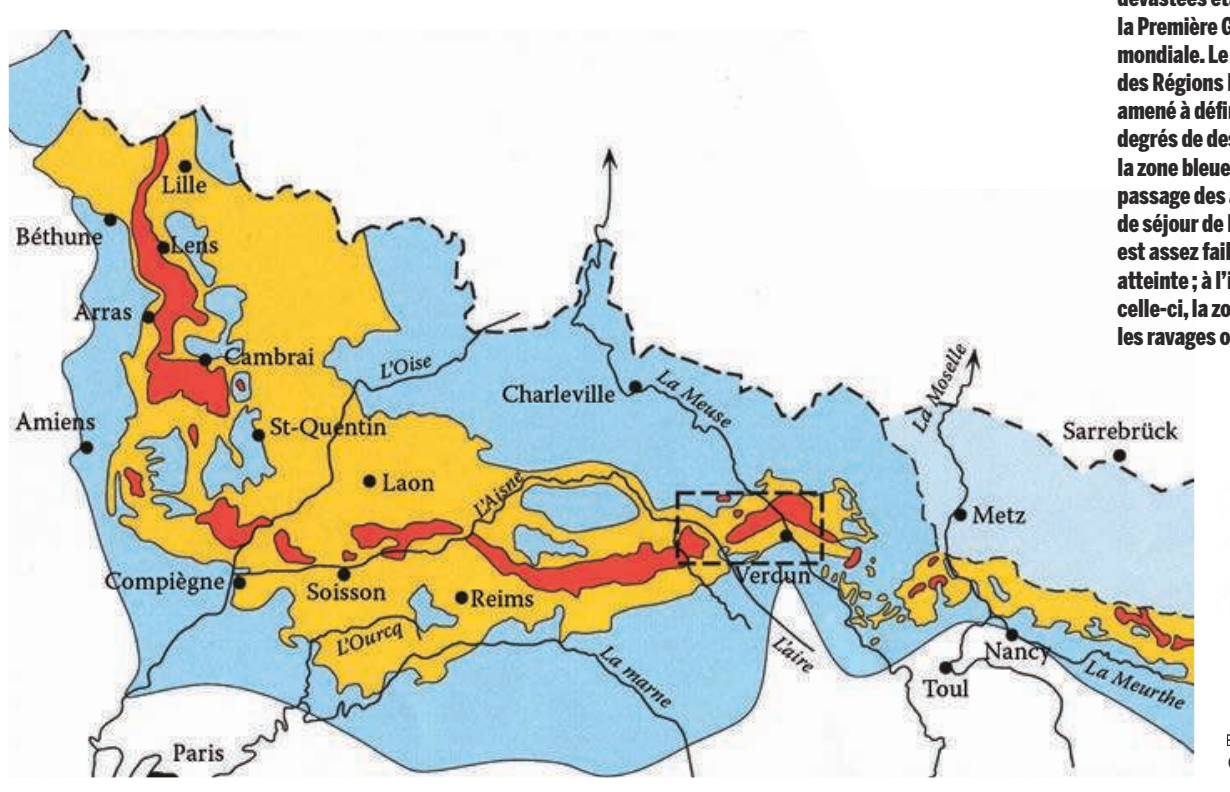

Carte des régions

dévastées établie après a Première Guerre miliale. Le ministère es Régions libérées sera (entés de destruction: ravages ont été beaucoup plus amples, correspond à la zone des opérations. Le sol, partiellement couvert d'ouvrages, y a été labouré de tranchées, de trous d'obus et criblé de projectiles, souvent non éclatés. La zone rouge est complètement détruite. C'est le lieu des grandes batailles. Le sol y a été retourné par les obus, souvent pilonné jusqu'au broyage total.
Le territoire de la Lorraine occupe une position particulièrement stratégique au cours des guerres mondiales. On y compte plusieurs batailles, la plus célèbre et la plus meurtrière étant celle de Verdun en 1916. De cette partie de l'histoire on relate souvent les faits militaires et rarement le sort des populations civiles. C'est le cas particulier des 13 villages détruits en Lorraine au cours de la Grande Guerre. Totalement dévastés, six d'entre eux ne seront jamais reconstruits. Ces villages jouissent pourtant d'un droit légal d'existence reconnu par l'État, fait historique d'exception souvent méconnu des milliers de visiteurs qui se rendent chaque année sur le champ de bataille. Administrativement, cela se traduit par la nomination par le préfet d'un maire chargé d'entretenir le lieu et de conserver les registres d'état civil. Sur le terrain, une plaque, une chapelle commémorative sont les seules marques qui signalent les anciennes habitations dont les ruines sont recouvertes par la végétation. Au-delà de la mémoire et des commémorations, ces espaces de recueillement sont des réserves patrimoniales de premier plan, qu'aujourd'hui les archéologues tentent d'explorer et de sauvegarder pour le futur.
Car depuis les années 1990, la réflexion d'une «archéologie du passé récent» s'est ouverte aux domaines variés de l'architecture et du patrimoine industriel et militaire. Les débats ont donné lieu à un rapport transmis au Centre national de la recherche archéologique. Ainsi, il est montré que l'histoire est faite d'une diversité de dynamiques développées à toutes les échelles temporelles et que pour l'étude de notre passé récent, la problématique porte sur la très courte amplitude, de quelques heures à quelques générations. Culturellement, les lieux qui ont été le théâtre d'événements exceptionnels ou tragiques doivent en garder la mémoire, à l'image du sol lunaire du champ de bataille de Verdun que vient de livrer LiDAR.

Sur ce sol mythique, il est possible aujourd'hui de caractériser des moments historiques et dramatiques. À l'image de Fleury où en l'espace de deux mois ce village «mort pour la France» a été pris et repris 16 fois par les belligérants. Le témoignage de ce passé «incommunicable» trouve sa traduction dans la densité des traces qui « disent» l'histoire. Aussi, à l'inverse des musées qui sacralisent le passé à travers quelques objets, la simple préservation in situ des vestiges et du mobilier permet de lire l'espace et donne un sens à la mémoire. 
La forêt, conservatoire

de la Grande Guerre

Après les quatre années de guerre, les terrains bouleversés du front lorrain sont de vastes espaces abandonnés. La reconstitution de toutes les terres est économiquement impossible, aussi par la loi du 17 avril 1919, l'ítat se rend acquéreur des territoires jugés irrécupérables qu'il délimite au crayon rouge sur une carte. Ainsi fut constituée la «zone rouge». Les terrains jugés les plus dangereux sont confiés à l'administration forestière afin d'être reboisés. La Meuse est unique dans l'histoire des zones rouges avec '79\% de terres reboisées. Ailleurs, dans le Pas-de-Calais ou dans la Somme, les zones rouges d'après guerre ont presque toutes été rétrocédées à l'agriculture lors des ventes des domaines. Comme la prairie, la forêt joue un rôle protecteur particulièrement efficace contre l'érosion des sols. ㅍlle permet la conservation de certaines constructions en terre et fossilise les vestiges en élévation. Ces espaces très conservateurs sont paradoxalement peu étudiés car difficile d'approche. Pourtant ces milieux sont privilégiés pour l'étude des anciennes activités artisanales tournées vers l'usage du bois, ou plus anciennement agropastorales avant l'existence de la forêt. Depuis peu, il s'avère que les forêt plantées après guerre sont également des réserves patrimoniales uniques. Ces forêts, vieilles d'à peine 100 ans, abritent toute la diversité des traces de l'occupation militaire mais également celles des civils : fermes et villages martyrs. À l'exemple du champ de bataille de Verdun qui compte 10000 ha de terrains bouleversés, dont neuf villages détruits par les bombardements.

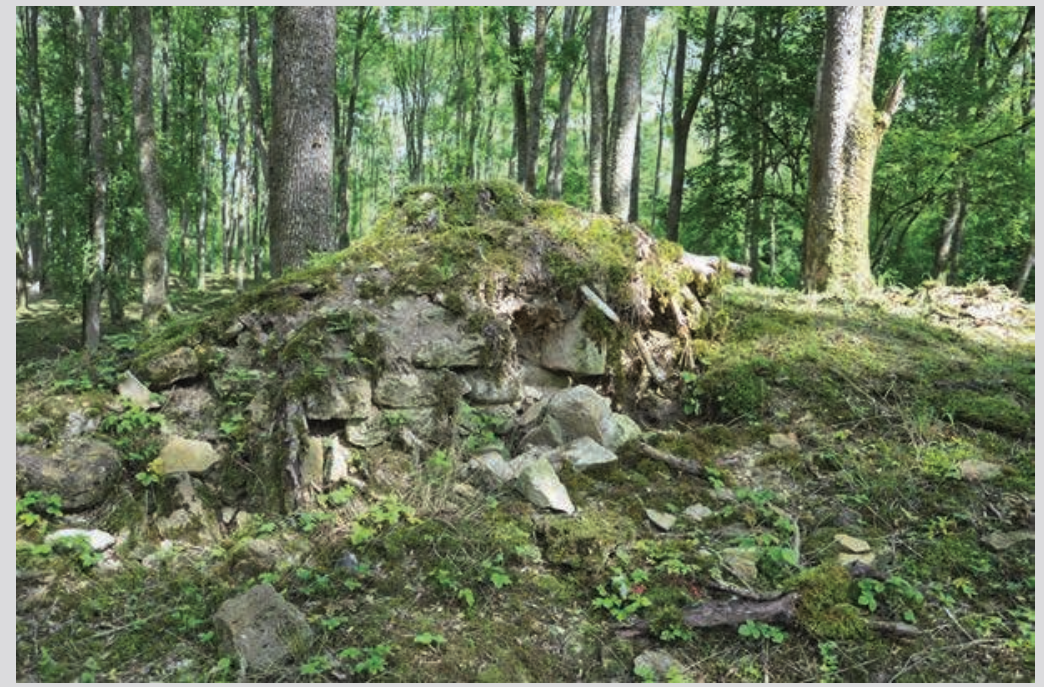

Bezonvaux (55). Les vestiges de guerre marquent durablement les milieux et forment des paysages à forte identité patrimoniale. En témoignent les ruines de maisons détruites par les combats et recouvertes par la forêt qui rappellent la mémoire des villages disparus.

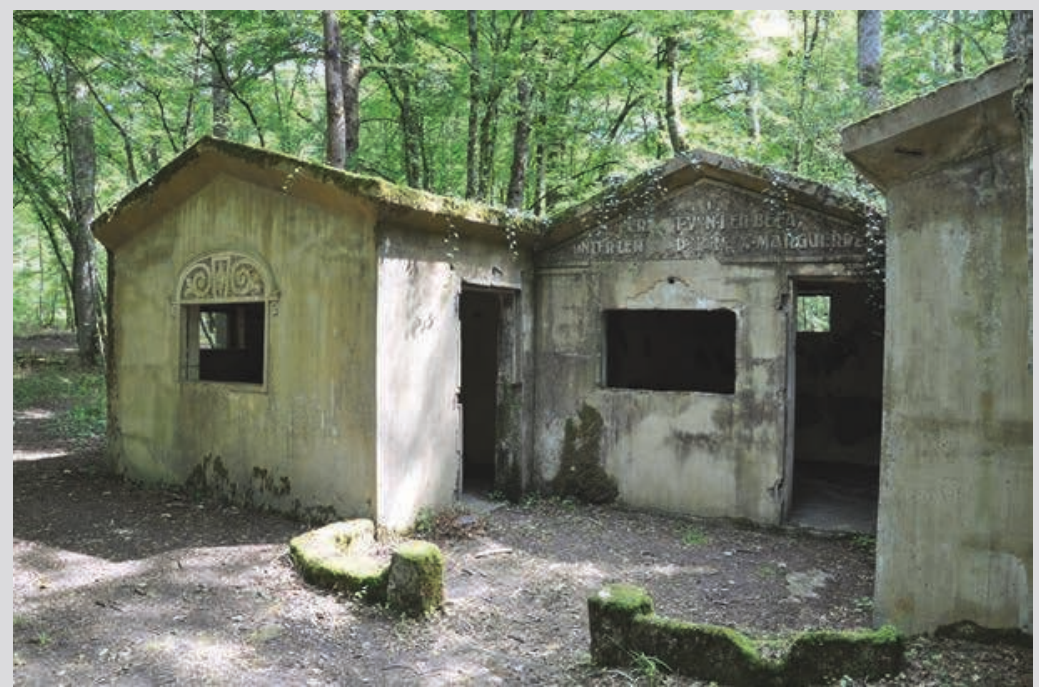

Loison « Camp Marguerre » (55). Les constructions en béton moulé, qui signent l'occupation allemande, sont plus résistantes aux bombardements et à l'érosion, à l'inverse des constructions maçonnées des occupations civiles.

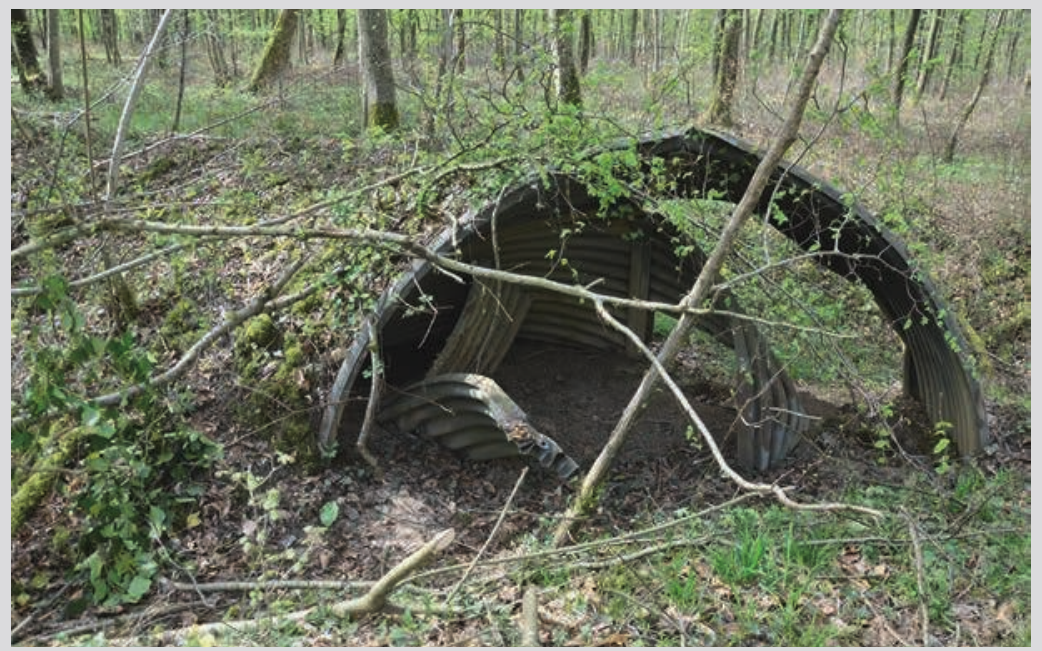

Forêt domaniale de Verdun (55). Dans la zone de combat, les abris et autres blockhaus français sont édifiés avec des matériaux fournis selon la disponibilité par les parcs du Génie. Sur l'arrière-front, dans les camps de repos, les soldats aménagent des abris avec des matériaux récupérés dans les ruines de bâtiments. 

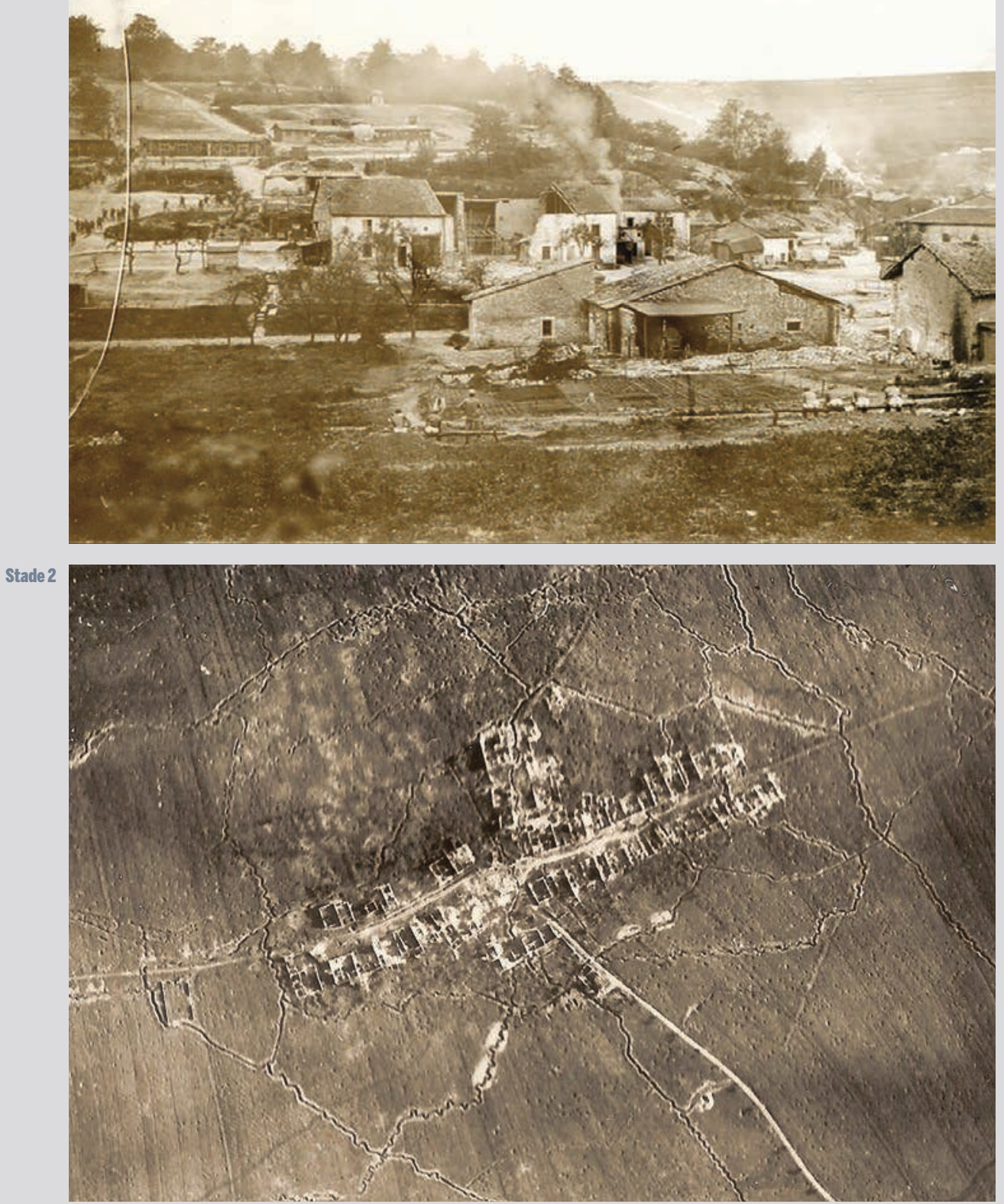

Stade 3

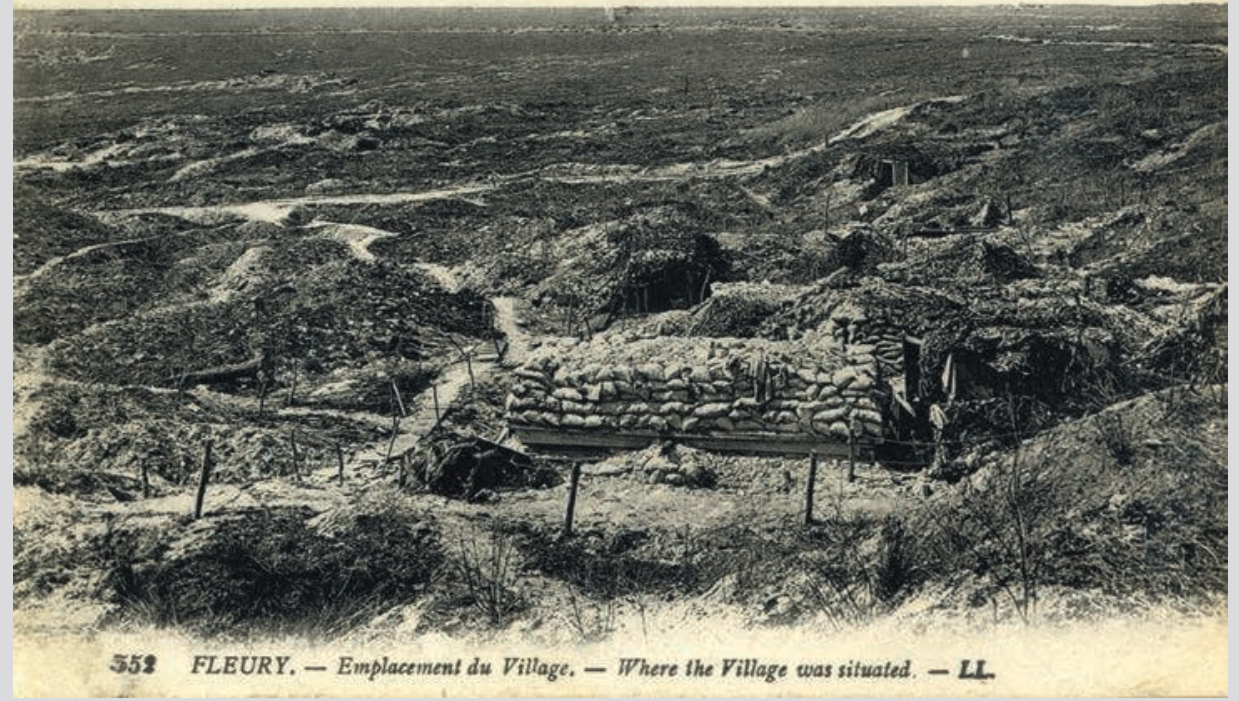




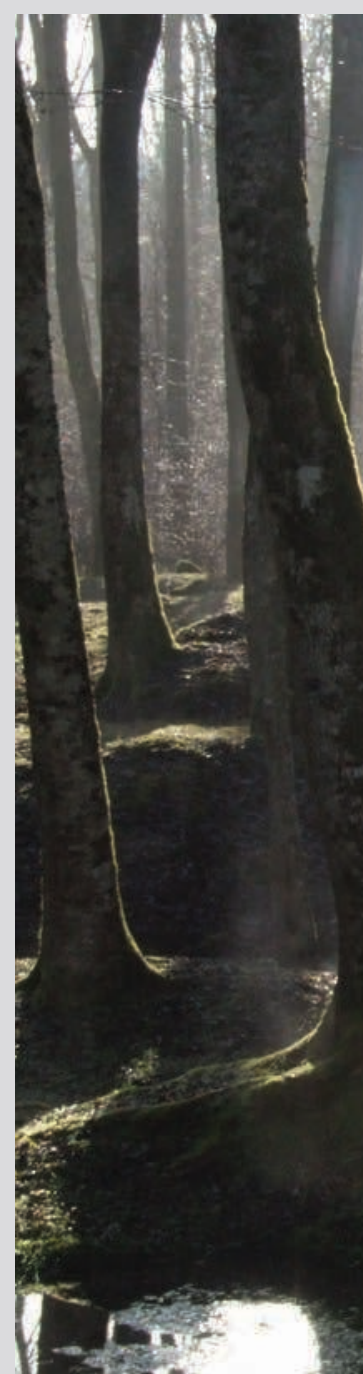

Stade 4

La construction des paysages de guerre, le polémofaciès des villages disparus La Première Guerre mondiale est un facteur déterminant dans l'évolution du paysage meusien. Cet épisode violent que l'on peut rattacher à l'ère de «l'Anthropocène " a modifié en profondeur et de manière irréversible l'environnement et la biogéographie de la zone de front. Pour la première fois dans l'histoire des sociétés, la mutation et la fossilisation d'un paysage se sont opérées en l'espace d'un demi-siècle à peine. D'une ruralité ancestrale avec ses villages, ses champs et bois communaux, on assiste, dès le début des hostilités, à l'apocalypse d'une terre dévastée, mutilée, crevassée. Quelques années plus tard, au terme du conflit, ce paysage, fortement dégradé par l'activité militaire, est reconquis par une végétation spontanée qui fixera les premiers polémoformes du champ de bataille (Amat, 1999, 2000). Vers 1920, la plantation en masse de résineux sur les anciennes terres agricoles et sur les villages détruits signe la fin de l'openfield. Aujourd'hui, dernier stade d'évolution, le paysage cicatrisé est modelé par les gestionnaires de la forêt et les aménagements touristiques. Les vestiges archéologiques, habituellement enfouis sous terre, le sont cette fois, mais sous un couvert végétal qui masque leur lisibilité.

et progressive d'un village lorrain de la zone rouge avant guerre.

Stade 2 : Mutation du paysage en polémofaciès (dynamique biogéographique sub-régressive). Dégradation rapide par l'action militaire, en témoigne cette photo aérienne du village détruit prise le 7 juin 1918 à une hauteur de $4000 \mathrm{~m}$ Stade 3 : Paysage fossilisé en polémofaciès abandonné (dynamique biogéographique progressive). Revégétalisation spontanée et/ou assistée tel le village martyr de Fleury-DevantDouaumont (55) qui, dés le sortir de la guerre, servira de carrière pour le réaménagement des routes.

Stade 4 : Paysage cicatriséen polémofaciès civil aménagé (dynamique biogéographique sub-régulée). La gestion sylvicole, les aménagements touristiques et la faune sauvage occupent les ruines des villages disparus. Ál'image de Bezonvaux où la forêt, tel un linceul vert, invite aujourd'hui à la sérénité et au recueillement. 


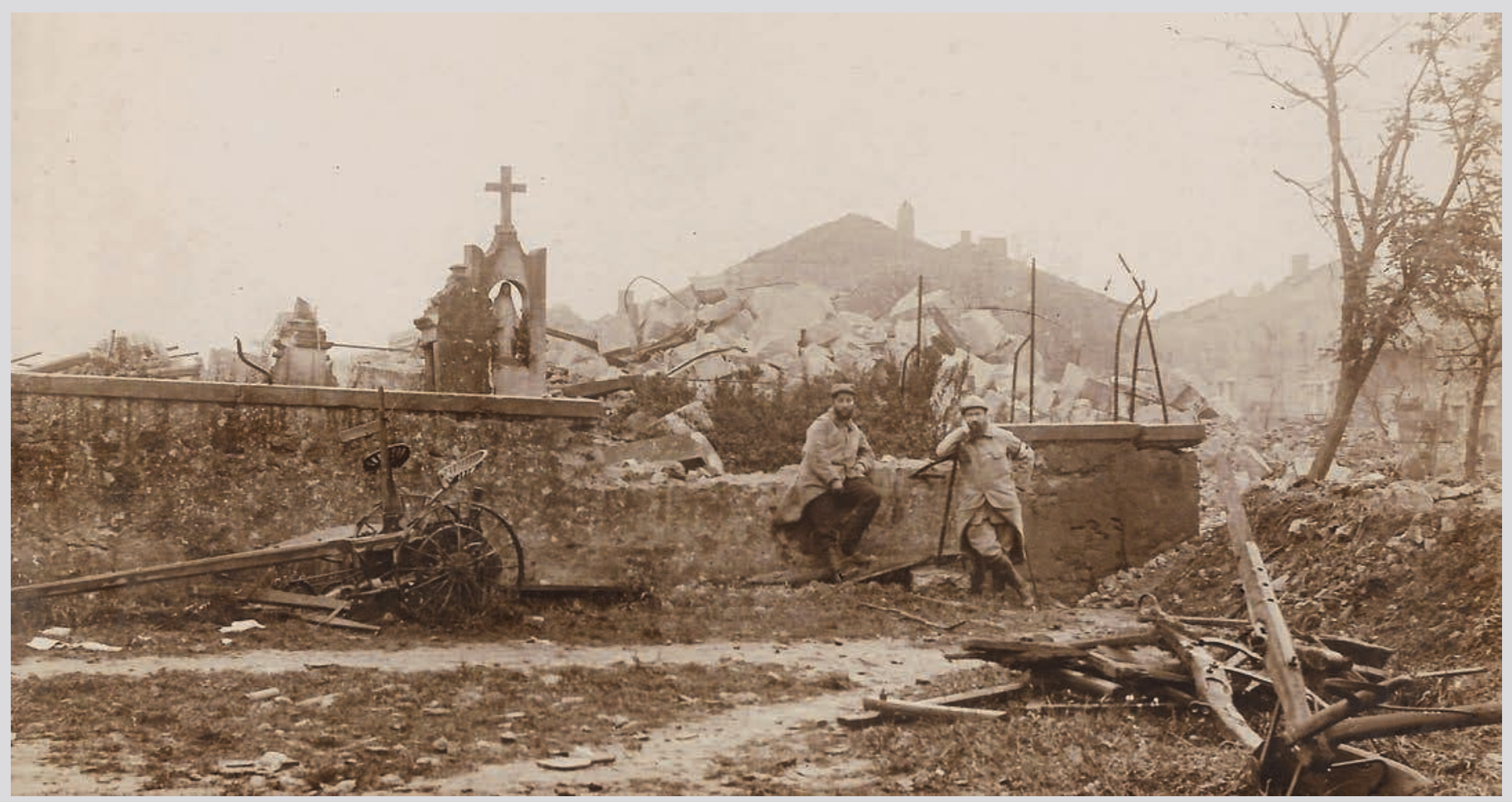

Photo des ruines du cimetière prise au milieu de l'année 1915.

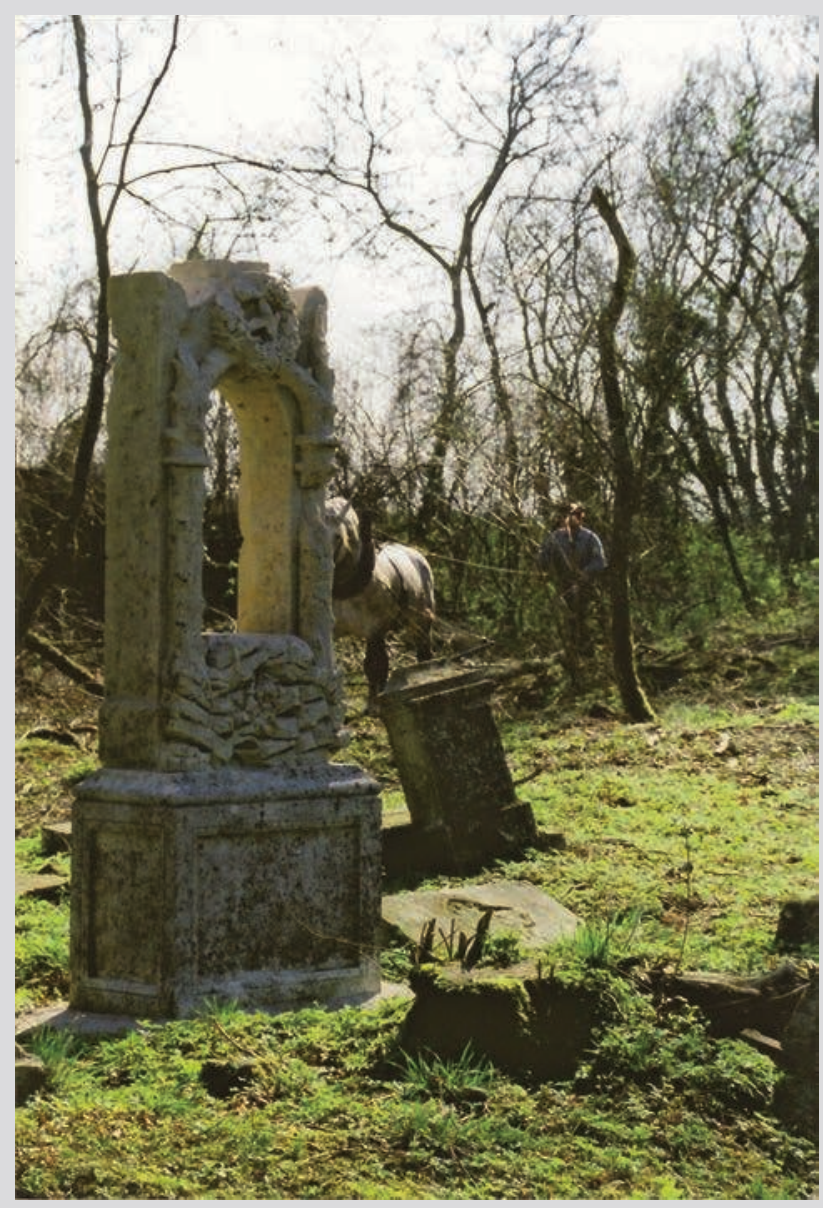

L'héritage bioculturel

du village détruit de Fey-en-Haye

Depuis longtemps, la prospection archéologique s'intéresse à la valeur indicatrice de la végétation pour détecter les vestiges. Les anomalies du tapis végétal trahissent la nature et la composition des sols anthropisés. Les sites en forêt de guerre illustrent particulièrement bien cette relation étroite entre la communauté végétale et l'occupation humaine. Ce sont des milieux privilégiés pour les études paléobotaniques qui visent à reconstituer l'histoire écologique des forêts (Steinbach, Husson, 2004). Malgrré les destructions massives qui ont bouleversé le sol en profondeur, les études botaniques menées ces dernières années ont permis d'identifier la présence de plantes rudérales (colonisatrices des ruines) ou obsidionales (introduites par l'occupation militaire). C'est le cas étonnant de «l'herbe aux yeux bleus", ce Sisyrinchium originaire de l'archipel des Bermudes qui aurait été propagé par les armées à l'occasion du fourrage fourni aux chevaux (Vernier, 2014). Fn 1998, 1'Office national des forêts participe au projet européen Iife «Héritage bioculturel des forêts ", portant sur la problématique des forêts de guerre. Plusieurs recherches historiques et scientifiques sont entreprises sur l'espace boisé du village détruit de Fey-en-Haye (Meurthe-etMoselle). Les relevés effectués durant une saison végétative ont permis de recenser un cortège de plantes issues des activités anciennes du village. Ces plantes dites « rélictuelles » ont perduré malgré les différentes phases de transformation du milieu lors des combats, de l'abandon du site et de son boisement après g̊uerre. Les résultats de cette étude sont aujourd'hui valorisés par un sentier botanique qui sillonne les rues du village détruit. On y rencontre : lilas, doronic orientale, houblon, groseillier, seringa, sumac de virginie, jonquille, sureau noir, petite pervenche, perce-neige et cytise. 
Carte LiDAR de la Forêt domaniale de Verdun (Meuse). Le fort de Douaumont "pilonné » par les bombardements est un symbole fort de la célèbre bataille de 1916 qui dura 300 jours et 300 nuits.

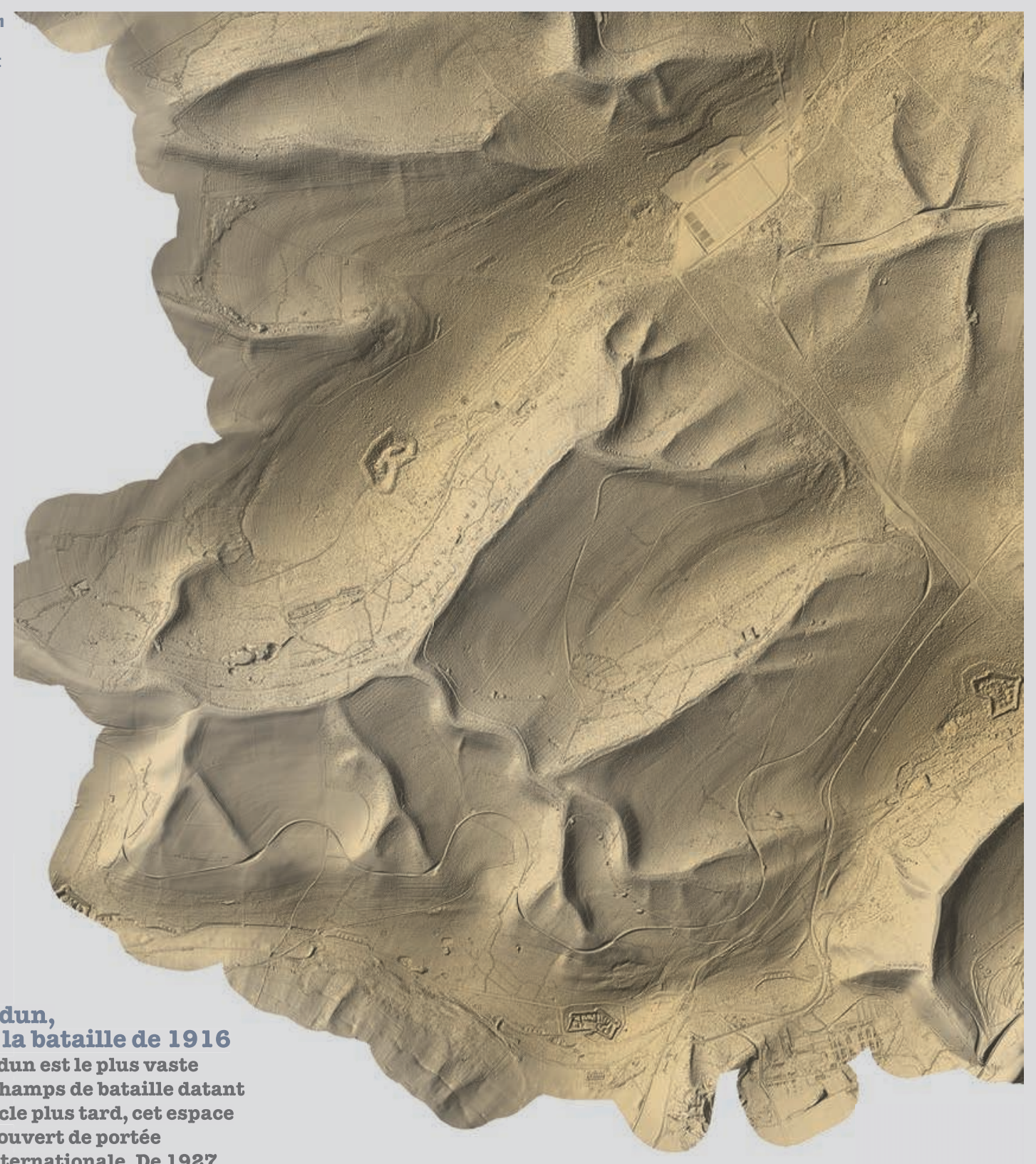

Les sacrifiés pour Verdun, valeur symbolique de la bataille de 1916 De référence mondiale, Verdun est le plus vaste et le plus authentique des champs de bataille datant de la Grande Guerre. Un siècle plus tard, cet espace naturel est un musée à ciel ouvert de portée historique et mémorielle internationale. De 1927 à 1934, plus de 6000 ha seront plantés en résineux en vue de reconstituer les sols qui, selon l'expression du président de la République Raymond Poincaré à l'occasion de sa visite sur le terrain le 21 août 1922, "recouvrent tel un vaste linceul l'espace des combats " (Laparra, 2006). Au cour de ce sanctuaire, 850 ha de site sont classés depuis 1967. Le périmètre, limité à la partie centrale du champ de bataille autour du fort de Daumont, est fréquenté par 200 à 300000 visiteurs chaque année. In 2006 , un projet d'extension du site classé est mis à l'étude, mais n'a pas abouti. Il devait prendre en compte les neuf villages détruits, les fermes en ruine ainsi que les secteurs historiques qui ont joué un rôle important dans l'affrontement. Ce site emblématique, qui s'inscrit dans les priorités du Grenelle de l'environnement, vient d'être labélisé sous le terme « Forêt d'exception » et fera dans les années à venir l'objet d'une attention particulière pour accueillir le public. Dans ce contexte, le ministère de la Culture et l'Office national des forêts viennent de réaliser la cartographie 3D du sol meurtri de la célèbre bataille, grâce une mission aérienne de télédétection LiDAR.

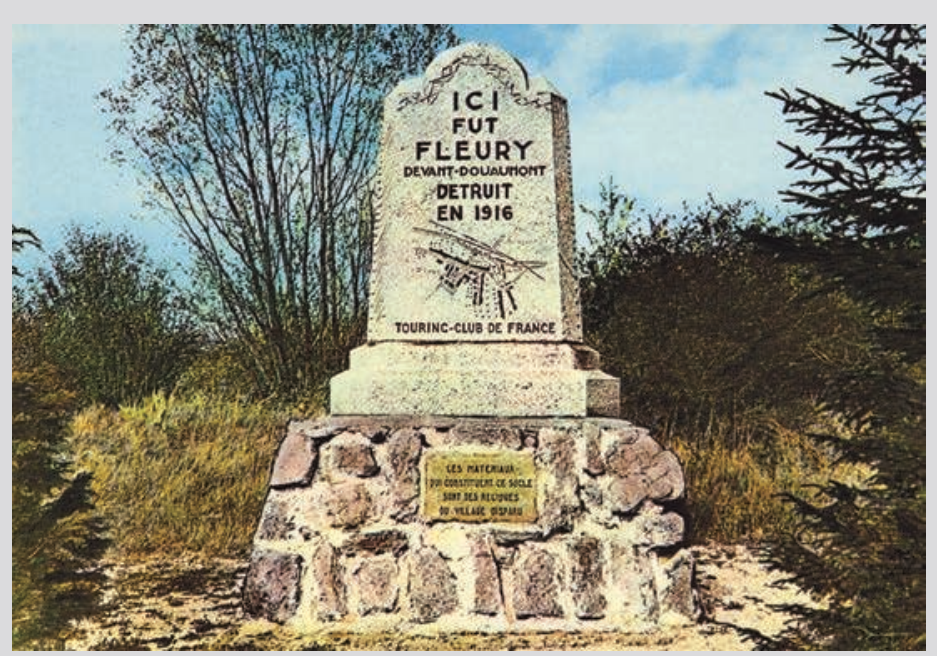

Au sortir de la guerre, les pèlerins sont déjà nombreux à venir se recueillir sur les ruines des villages martyrs. En 1918, la petite commune normande "Allemagne » a changé son nom pour devenir Fleury-sur-Orne en l'honneur de ce village « mort pour la France ». 


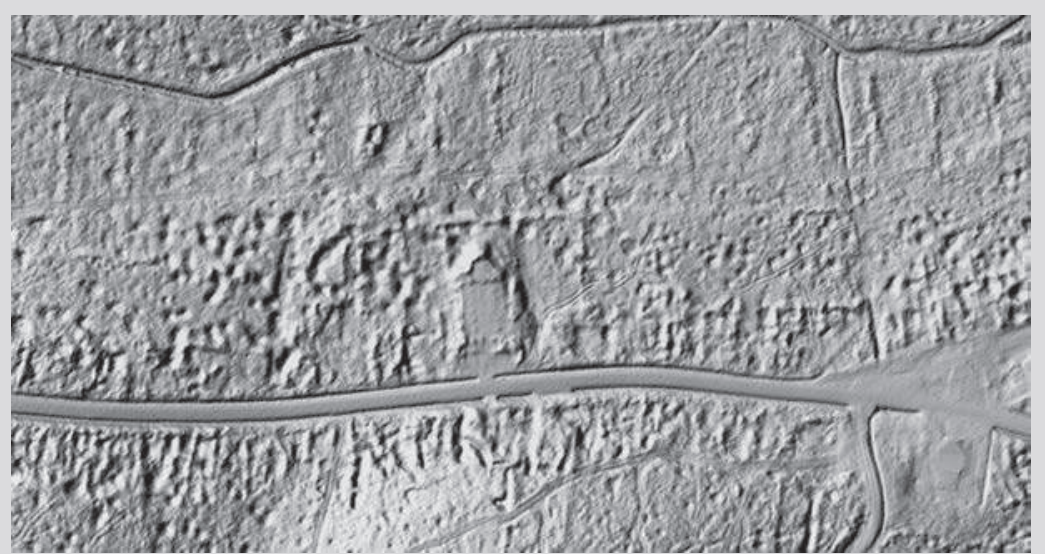

Le traitement de la visualisation du MNT par ombrage permet de modifier l'angle zénithal et l'azimut de la lumière, afin de mettre en valeur les reflets en creux.

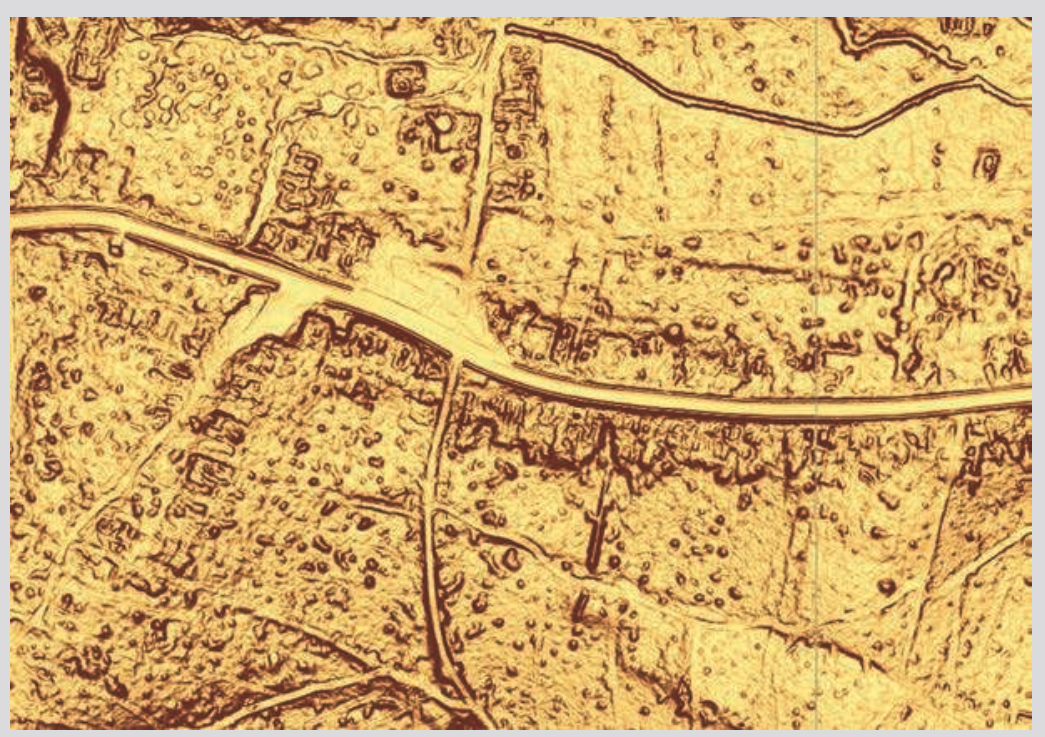

Le traitement de la visualisation du MNT par la pente (cinq classes de valeur) favorise la lecture des ruptures de profils topographiques, comme les tranchées.

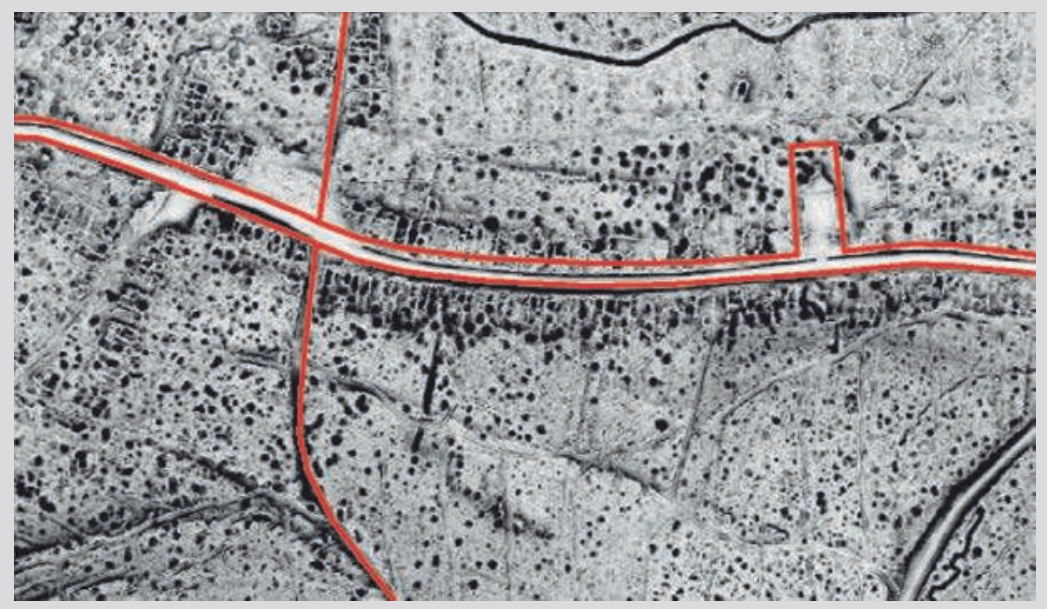

Le traitement de la visualisation du MNT par SkyView Factor permet de mettre en valeur les indices les moins éclairés, comme ici les polémoformes des pièces d'habitation.
Les vestiges de ǵuerre sous l'œil du laser Outil de prédilection des archéologues ces dernières années, cette avancée technologique a fortement contribué à renseigner la carte archéologique dans les espaces boisés jusqu'alors peu appréhendés. Cette technique laser permet de s'affranchir du bruit atmosphérique et surtout de la végétation, pour produire une image 3D de la surface terrestre. $\mathrm{Fn} 2013$, une mission LiDAR a été effectuée dans le cadre du projet « Verdun 14/18, Forêt d'exception ". Alors qu'on ne connaissait que les parties fortifiées du champ de bataille, cette nouvelle technologie a révélé en quelques jours la totalité des vestiges sur près de 12000 ha. Outre les réseaux de tranchées et les impacts de bombardement, on y observe toutes les traces de vie des soldats parfois associées à celles des villages fantômes. Les premières données exploitées à ce jour font état d'un territoire archéologique semblet-il unique en Furope, compte tenu de l'état de conservation des structures et de leur bonne lisibilité. Dans le cadre du centenaire de la Grande Guerre, un projet de thèse, mené en partenariat entre l'UIMR 8586 Prodig (université Paris-Diderot) et le Service régional de l'archéologie de Lorraine, porte sur «l'archéogéomorphologie du champ de bataille de Verdun".

Archéogéomorphologie du champ de bataille de Verdun

L'aspect géomorphologique et environnemental traitera des impacts de la guerre sur la transformation des sols. Il est question de produire une typologie inédite des formes des reliefs anthropogéniques créées par le confilit et d'analyser la pédogenèse liée à la désagrégation des obus enfouis dans le sol. Les occupations civiles des villages disparus est au coeur de l'étude. Ici, le village d'Ornes. 

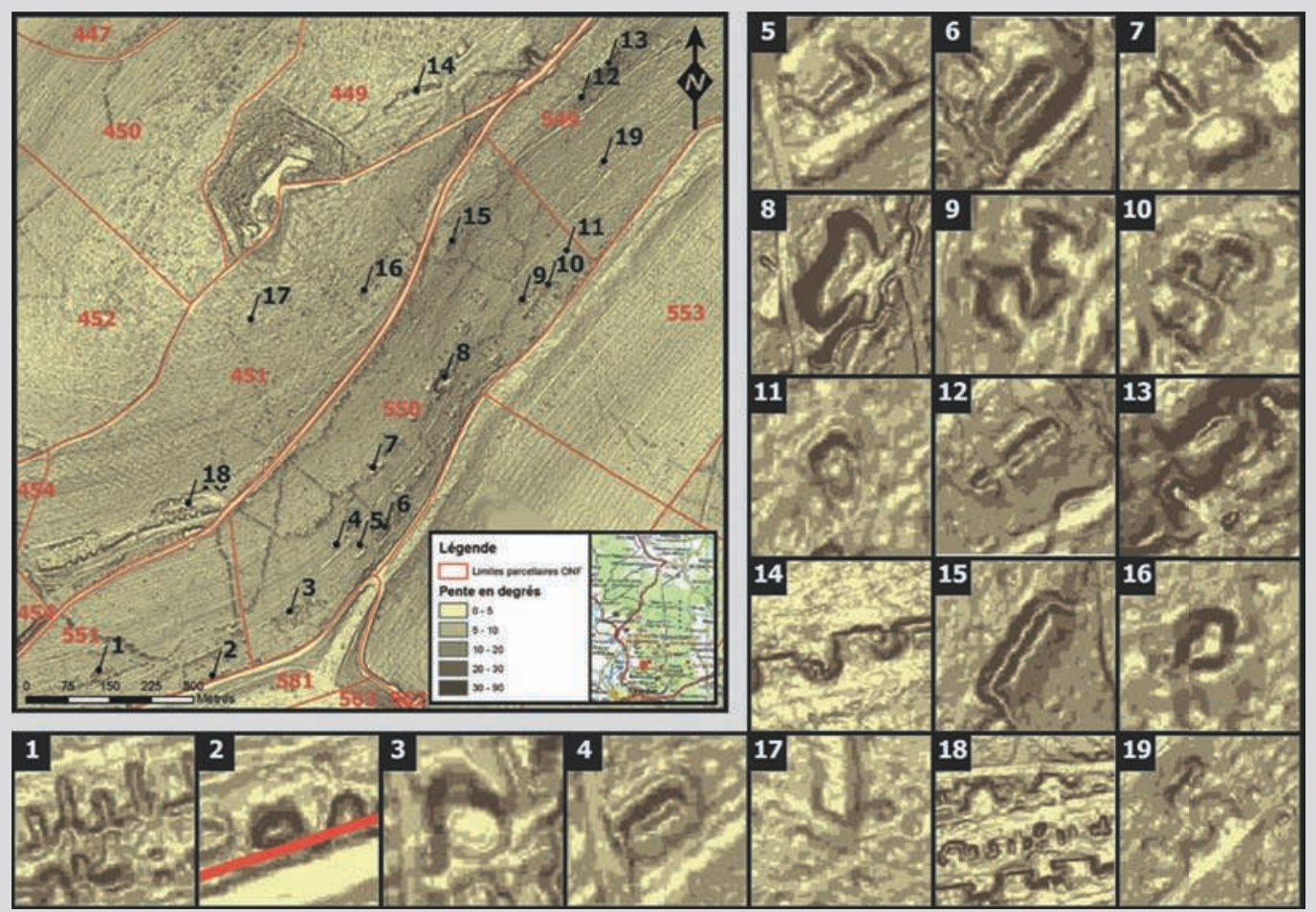

L'innovation méthodologique du traitement de l'imagerie LiDAR, combinée à la géomatique, permettront de construire un Système d'information géographique du champ de bataille. Accompagné de bases de données multiples (descriptives, iconographiques, documentaires) ce SIG permettra d'enrichir l'analyse spatiale pour produire des cartes interprétatives inédites et visualiser l'évolution matérielle du conflit. Ici, Forêt domaniale de Verdun, Bois d'Ormont (Meuse).
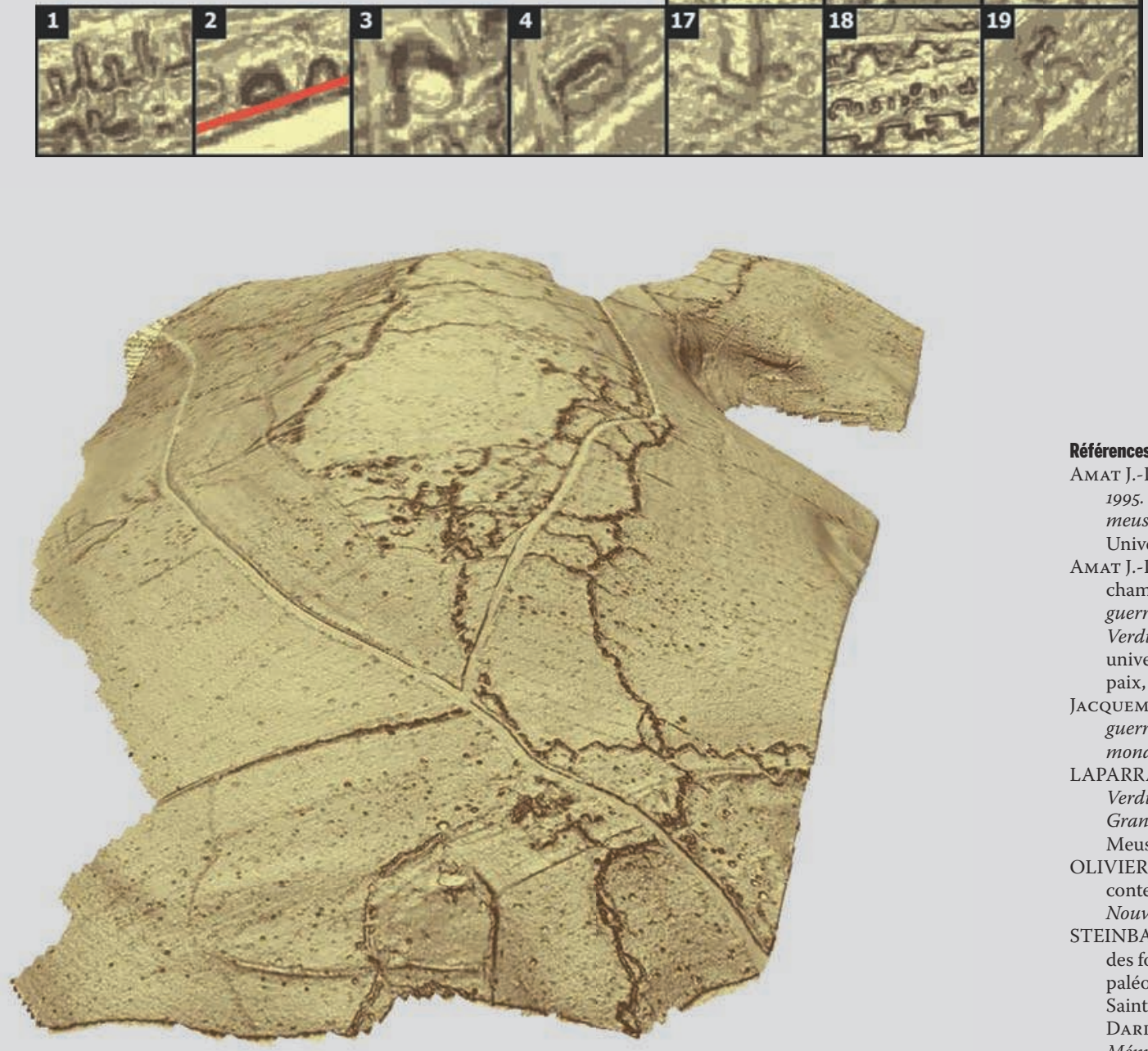

\section{Références bibliographiques}

АмAT J.-P., 1999, La Forêt entre guerres et paix, 1870 1995. Étude de biogéographie historique sur l'arc meusien de l'Argonne à la Woëvre, Thèse d'État, Université de Lille, 1285 p.

АмАт I.-P., 2000, «Nettoyer, restaurer, réaffecter le champ de bataille: la zone rouge », in Finir la guerre, Actes du colloque «Histoire de Péronne», Verdun, 12-13 novembre 1999, Nancy, Presses universitaires de Nancy (coll. Les cahiers de la paix, 7), p. 103-132.

JACQUEMOT S., LeGENDRE J.-P. (DIR), 2012, Vestiges de guerres en Lorraine. Le patrimoine des conflits mondiaux, Metz, éd. Serpenoise, $231 \mathrm{p}$.

LAPARRA J., LAPARRA J.-C., 2006, Sacrifié pour Verdun. Bezonvaux, village détruit pendant la Grande Guerre, Verdun, Connaissance de la Meuse (coll. Connaissance de la Meuse), 157 p.

OLIVIER L.,1997, « L'archéologie du passé contemporain: enjeux et perspectives", Les Nouvelles de l'archéologie, 70, p. 7-14.

STEINBACH F., HUSSON I.-P., 2004, «La mémoire des forêts. Palimpsestes et héritages des paléopaysages dans les massifs du saillant de Saint-Mihiel », in Dupouey J.-L., Dambrine É Dardignac C., Georges-Leroy M. (Dir.), $L a$ Mémoire des forêts, Actes du colloque Forêt, archéologie et environnement, Velaine-en-Haye, 14-16 décembre 2004, Paris, ONF/Inra/Drac Lorraine, p. 285-294.

Grâce aux potentialités offertes par la télédétection LiDAR, il est possible d' identifier, de manière précise et géoréférencée, les vestiges et les formes de reliefs en s'exonérant d'aller sur le terrain. Forêt domaniale de Verdun (Meuse). Représentation 3D d'une zone du conflit mise en évidence par la télédétection LiDAR.

VERNIER F, 2014, Plantes obsidionales. L'étonnante histoire des plantes propagées par les armées en Lorraine, Strasbourg, Vent D’Est, $192 \mathrm{p}$ 